\title{
Information system success and knowledge grid integration in facilitating knowledge sharing among big data community
}

\begin{abstract}
Nowadays many domains interested to use big data to improve their decision making, strategic planning, and productivity while communication infrastructure supports various applications for users to share their resources, ideas or experiences. However, accessing, managing, analyzing, and using the rapidly expanding big data had raised challenges especially because of dispersed and heterogeneous nature of data. This review aimed to identify the factors to influence facilitating knowledge sharing among big data community from user satisfaction aspect and the way knowledge grid effect big data sharing by Delone and Maclean model for information system success. The research analysis and results revealed three features of knowledge grid which may influence information and system quality which defined as main factors affect knowledge sharing among the community from member's aspect. It also demonstrates seven factors to measure the facilitating knowledge sharing from community member's satisfaction view and quantity of knowledge sharing. Finally, the review established an initial conceptual model for facilitating knowledge sharing among big data which in follow up research will review by domain experts.
\end{abstract}

Keyword: Knowledge sharing; Knowledge grid; Big data community 